\title{
A Rare Case Report of Granulomatous Inflammation of Gall Bladder with Review of Literature
}

\author{
Dr. Bhavna Valecha* \\ Resident, Department of Pathology, BPS GMC for Women, Ganaur - Gohana Rd, Khanpur Kalan, Haryana 131305, India
}

DOI: $\underline{10.36348 / \text { sjpm.2019.v04i11.013 }}$

| Received: 18.11.2019| Accepted: 25.11.2019| Published: 28.11 .2019

*Corresponding author: Dr. Bhavna Valecha

\section{Abstract}

Introduction: Gallbladder tuberculosis (GBTB) is an exceedingly rare finding presenting as calculous or acalculous cholecystitis. A correct, preoperative diagnosis of GBTB is difficult, and the literature review highlights the fact that most cases are diagnosed postcholecystectomy or at postmortem. Discussion: Mycobacterium tuberculosis can affect any part of the gastrointestinal tract from the mouth to the anal canal along with the other organs of the peritoneal cavity, but the gall bladder is unlikely to be primarily involved. The incidence of localization of the tubercular bacilli in the gall bladder is uncommon as the intact gall bladder mucosa is resistant to the bacterium because of the concentrated bile acids present in the organ. The first case of gallbladder tuberculosis (GBTB) was reported in 1870 by Gaucher, and till now, less than 120 cases have been reported in the English medical literature [4, 5].

Keywords: Granulomatous Inflammation, Gall Bladder tuberculosis, postmortem.

Copyright @ 2019: This is an open-access article distributed under the terms of the Creative Commons Attribution license which permits unrestricted use, distribution, and reproduction in any medium for non-commercial use (NonCommercial, or CC-BY-NC) provided the original author and sources are credited.

\section{INTRODUCTION}

Cholelithiasis and associated cholecystitis of the gallbladder (GB) is a very common diagnosis made in patients presenting with right upper quadrant abdominal pain. A gallstone occurs as a secondary complication of GB infection. The infecting organisms are of many types, and mycobacterium as the causative organism is rarely found and suspected and much less frequently proven. Isolated tuberculosis (TB) of the GB is extremely rare, and the diagnosis of this disease is almost always overlooked, unless there is a high index of suspicion.

\section{CASE REPORT}

A 37 year female Wall thickness was $3 \mathrm{~cm}$, multiple mixed stone seen in the lumen, gallbladder size $4.5 \times 2.5$.

Another case of 55 year old male with wall thickness $3 \mathrm{~cm}$ and gall bladder size of $9 \times 2.5 \mathrm{~cm}$ and multiple mixed stone inside.

Both the patients presented with a history of right upper abdominal pain associated with episodes of vomiting every 2-3 weeks for the past 1 year. No history of fever, jaundice, loss of appetite, or weight loss was reported. Physical examination of the patient showed no abnormal findings.

There was no evidence of peripheral lymphadenopathy or hepatosplenomegaly. After taking a detailed clinical history, ultrasonography of the abdomen revealed a thickened gall bladder wall with multiple gall stones, leading to a diagnosis of chronic cholecystitis with cholelithiasis.

An abdominal ultrasound revealed a distended GB with multiple calculi and normal wall thickness.

X-ray of the chest and all other hematological parameters were also normal. The patient underwent a routine laparoscopic cholecystectomy which was uneventful.

GROSS -Intraoperatively, the GB was distended with multiple calculi, the cystic duct was short and dilated, and dense adhesions were seen between the duodenum and the GB wall. The liver, the common bile duct, and the rest of the bowel were grossly normal.

Microscopy- Histological examination of the Gall Bladder revealed a mucosa showing focal ulcerations and dense chronic inflammation in the subepithelium with few, scattered, noncaseating, 
epithelioid cell granulomas with giant cells although acid-fast stain was negative. The surgery was terminated and the histopathology closely followed.

Granulomatous inflammation of gall bladder showing ill formed granulomas with Langhans' giant cells with epithioloid cells and lymphocytes was seen.

Gastrointestinal tuberculosis usually presents as involvement of the peritoneum, intestine, or the lymph nodes, and isolated tuberculosis of the gall bladder is extremely rare with only about 50 cases reported in literature [1].

The rarity of the disease involving the gall bladder could be attributed to the fact that bile is thought to have an inhibitory effect on the gall bladder mucosa for the development of tuberculosis.

Cholelithiasis and cystic duct obstruction are considered important factors for its development [2, 3] as seen in our patient.

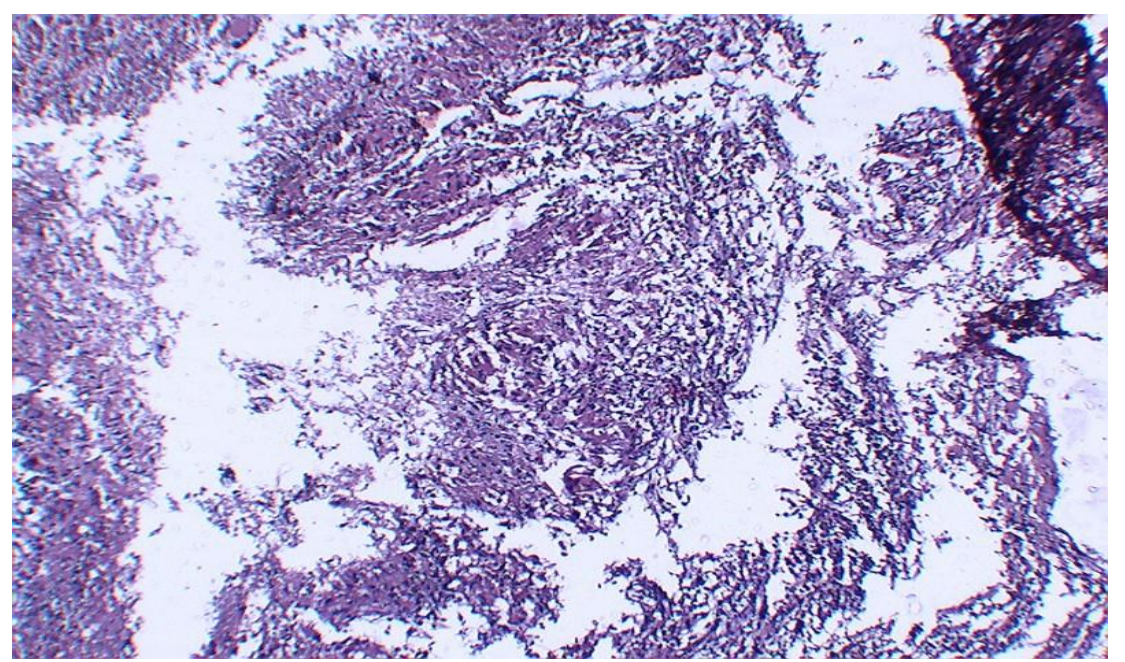

ILL Formed Granulomas and Giant Cells (Granulomatous Inflammation) 100X, H\&E

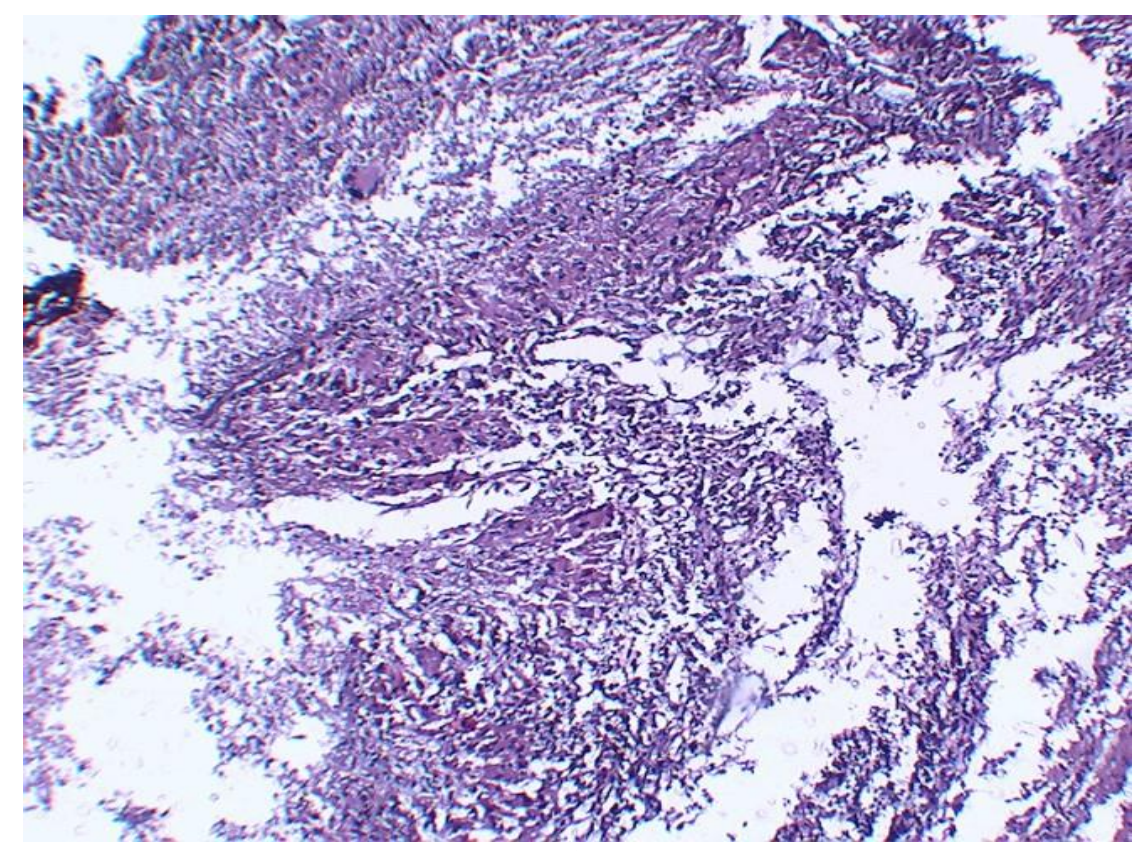

ILL Formed Granulomas and Giant Cells (Granulomatous Inflammation) 100X, H\&E

\section{DISCUSSION}

GBTB represents $1 \%$ of the abdominal TB cases [4]. Cholelithiasis and cystic duct obstruction are considered as the most important factors in the development of GBTB, with cholelithiasis being present in $70 \%$ of the cases [6].
GB mucosa is highly resistant to tubercular infection, possibly because of the inhibitory effect of the concentrated bile acids in the GB lumen and the high alkalinity of the bile [7].

GBTB can be manifested by a relatively nonspecific clinical presentation (abdominal pain, weight loss, low-grade fever, anorexia, vomiting, and 
abdominal mass), as seen in our case. It occurs most commonly in women over 30 years of age [2], Gallbladder tuberculosis (GT), especially the isolated GT, is an exceedingly rare disease entity [8]. GT often occurs along with other intra-abdomen tuberculoses, which reach the gallbladder via the lymphatics or bloodstream, and GT mostly happens in women over the age of $30[9,2]$. Around $70 \%$ of GT cases are accompanied by gallstones [10]

\section{CONCLUSION}

In our cases stain for acid fast bacilli was negative but the most common cause of granulomatous inflammation of gall bladder is tuberculosis.

\section{REFERENCES}

1. Yu, R., \& Liu, Y. (2002). Gallbladder tuberculosis: case report. Chinese medical journal, 115(8), 1259-1261.

2. Abu-Zidan, F. M., \& Zayat, I. (1999). Gallbladder tuberculosis (case report and review of the literature). Hepato-gastroenterology, 46(29), 2804-2806.

3. Rouas, L., Mansouri, F., Jahid, A., Zouaidia, F., Saidi, H., Nabih, N., ... \& Elhachimi, A. (2003). Gallbladder tuberculosis associated with cholelithiasis. Revue medicale de Liege, 58(12), 757-760.
4. Xu, X. F., Yu, R. S., Qiu, L. L., Shen, J., Dong, F., \& Chen, Y. (2011). Gallbladder tuberculosis: CT findings with histopathologic correlation. Korean journal of radiology, 12(2), 196-202.

5. Kumar, K., Ayub, M., Kumar, M., Keswani, N. K., \& Shukla, H. S. (2000). Tuberculosis of the gallbladder. HPB Surgery, 11(6), 401-404.

6. Lakhey, P., Shrestha, M. L., Sayami, G., \& Khakurel, M. P. (2006). Gall bladder tuberculosis: A rare entity. Journal of Institute of Medicine, 28(3), 53-54.

7. Kapoor, S., Sewkani, A., Naik, S., Sharma, S., Jain, A., \& Varshney, S. (2006). Myriad presentations of gall bladder tuberculosis. Indian journal of gastroenterology: official journal of the Indian Society of Gastroenterology, 25(2), 103104.

8. Rejab, H., Guirat, A., Ellouze, S., Trigui, A., Mizouni, A., Triki, H., ... \& Beyrouti, M. I. (2013). Primitive gallbladder tuberculosis: a case report with review of the literature. Annali italiani di chirurgia, 84(ePub), 1-3.

9. Collierand, N. A., \& Blumgart, L. H. (1994). Tumours of gall bladder, in Surgery of the Liver and Biliary Tract, L. H. Blumgart, Ed., 55-56, Churchill Livingstone, New York, NY, USA, $2^{\text {nd }}$ edition.

10. Yuand, R., \& Liu, Y. (2002). Gallbladder tuberculosis: case report. Chinese Medical Journal, 115(8):1259-1261. 